\title{
A Search for Instantons at HERA
}

\author{
Birger Koblitz for the H1 collaboration ${ }^{\dagger}$
}

November 13, 2018

\begin{abstract}
A search for QCD instanton $(I)$ induced events in deep-inelastic scattering (DIS) at HERA is presented in the kinematic range of low $x_{\mathrm{Bj}}$ and low $Q^{2}$. After cutting into three characteristic variables for $I$ induced events yielding a maximum suppression of standard DIS background to the $0.1 \%$ level while still preserving $10 \%$ of the I-induced events, 549 data events are found while $363_{-26}^{+22}(\mathrm{CDM})$ and $435_{-22}^{+36}$ (MEPS) standard DIS events are expected. More events than expected by the standard DIS Monte Carlo models are found in the data. However, the systematic uncertainty between the two different models is of the order of the expected signal, so that a discovery of instantons can not be claimed. An outlook is given on the prospect to search for QCD instanton events using a discriminant based on range searching in the kinematical region $Q^{2} \gtrsim 100 \mathrm{GeV}^{2}$ where the $I$-theory makes safer predictions and the QCD Monte Carlos are expected to better describe the inclusive data.
\end{abstract}

\section{Introduction}

Instantons [1], 2] are tunnelling phenomena between topologically different vacuum states present in non abelian gauge theories which can not be described by perturbation theory. In the case of QCD, instantons $(I)$ induce hard processes violating chirality. Deep-inelastic scattering (DIS) offers a unique opportunity [3] to discover processes induced by QCD instantons because a sizeable rate is predicted within "instanton-perturbation theory"

*Max Planck Institut für Physik, c/o DESY FH1, Notkestr. 85, 22607 Hamburg, koblitz@mail.desy.de

${ }^{\dagger}$ invited talk given at the Ringberg Workshop on HERA Physics on June 19th, 2001 


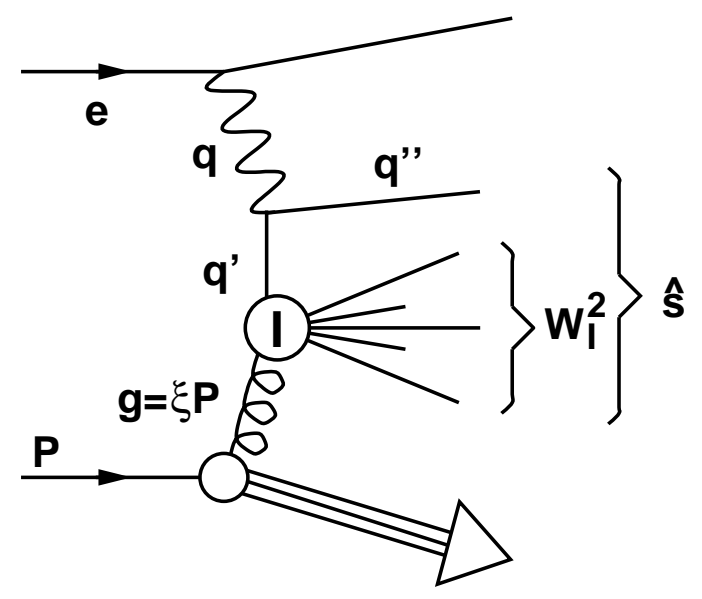

DIS variables:

$Q^{2}=-q^{2}$

$x=Q^{2} /(2 P \cdot q)$

$\hat{s}=(q+g)^{2}$

Variables of $I$-subprocess:

$Q^{\prime 2}=-q^{\prime 2}=-\left(q-q^{\prime \prime}\right)^{2}$

$x^{\prime}=Q^{\prime 2} /\left(2 g \cdot q^{\prime}\right)$

$W_{\mathrm{I}}^{2}=\left(q^{\prime}+g\right)^{2}=Q^{\prime 2}\left(1-x^{\prime}\right) / x^{\prime}$

Figure 1: Sketch of a boson gluon fusion process inducing instanton transitions. A virtual photon (with 4-momentum $q$ ) emitted by the incoming electron fluctuates into a $q-\bar{q}$-pair, one of which (with 4 -momentum $q^{\prime}$ ) fuses with a gluon $(g)$ out of the proton while the other (current quark) forms a hard jet. The $I$-subprocess is characterized by the negative 4-momentum squared $Q^{\prime 2}=-q^{\prime 2}$ of the in-coming quark and $x^{\prime}$ which is defined in analogy to the standard DIS variable $x_{\mathrm{Bj}}$.

[4, 5, 6], and instanton events exhibit a characteristic final state signature [7, 81.

At the HERA machine, which collides $27.5 \mathrm{GeV}$ positrons on $820 \mathrm{GeV}$ protons, the predicted cross section is large enough to make an experimental observation possible, although the expected signal is still small compared to the standard DIS background. Therefore, finding discriminating observables based on the expected "fire-ball" like topology of $I$-induced events producing a large number of hadrons and combining these variables to a powerful discriminant is the key ingredient for a successful search analysis.

In contrast to the first pioneering studies which were concentrated on relatively few variables characterizing $I$-induced events [9, 10, 11], the analysis by the $\mathrm{H1}$ collaboration [29, 30] which is presented here uses dedicated variables and optimized cuts.

\section{Instanton-induced processes in DIS}

Instanton processes in DIS are dominantly induced by photon gluon fusion as sketched in figure [1, where a photon emitted by the incoming electron 
splits into a $q-\bar{q}$-pair. One of these quarks $\left(q^{\prime}\right)$ enters into the $I$-subprocess. The basic reaction in this subprocess is

$$
\gamma^{*}+g \Rightarrow \sum_{\text {flavours }}\left(\bar{q}_{R}+q_{R}\right)+n_{g} g
$$

where $q_{R}\left(\bar{q}_{R}\right)$ denote right handed quarks (anti-quarks) and $g$ gluons. In every $I$-induced event, one quark anti-quark pair of all $n_{f}$ kinematically accessible flavours is produced. Charm and bottom quarks can in principle be produced, but their cross section is strongly suppressed. Chirality is violated by these events with $\Delta \chi=2 n_{f}$. Anti-Instantons also contribute to the cross section in the same manner, but here only left handed quarks are in the final state. The quarks are emitted isotropically together with a mean of $\left\langle n_{g}\right\rangle \approx \mathcal{O}\left(1 / \alpha_{s}\right) \approx 3$ gluons and fragment into a densely populated band of hadrons with a width of about 1.1 units of $\eta$. They are homogeneously distributed in azimuth. This band together with the relatively hard jet originating from the current quark form the characteristic final state of $I$-induced events. Since in each $I$-event a pair of strange quarks is produced, in addition an increase in the amount of strange hadrons is expected in the band region.

The actual number of produced hadrons heavily depends on the squared centre of mass energy $W_{I}^{2}=\left(q^{\prime}+\xi P\right)^{2}$ of the $I$-system. It can be expressed in terms of $Q^{\prime 2}$ and $x^{\prime}$ by $W_{I}^{2}=Q^{\prime 2}\left(1-x^{\prime}\right) / x^{\prime}$, where $Q^{\prime 2}$ is the negative four momentum vector squared of the quark entering the $I$-subprocess and $x^{\prime}$ is a variable describing this process in analogy to the Bjorken scaling variable $x_{\mathrm{Bj}}$ in DIS. The instanton cross section can be safely calculated [5] in "instanton perturbation theory" only for large values of $Q^{\prime 2}$ and $x^{\prime}$. Towards lower values the cross section calculated perturbatively steeply increases. Matching the instanton size and distance distributions in the QCD vacuum, which are fundamental properties of instantons, to high quality lattice calculations allows to derive lower boundaries $Q^{\prime 2}>113 \mathrm{GeV}^{2}, x^{\prime}>0.35$ above which the perturbative calculations are applicable.

The most recent calculations of the cross section for $I$-induced events at the HERA collider requires an additional cut on $Q^{2} \geq Q^{\prime 2} \approx 113 \mathrm{GeV}^{2}$ to further reduce theoretical uncertainties due to non-planar diagrams, which are not present in the calculation. In the kinematic region defined by the cuts required by the perturbative ansatz $x^{\prime}>0.35, Q^{2} \geq Q^{\prime 2} \geq 113 \mathrm{GeV}^{2}$ and general additional cuts $x>10^{-3}$ and $0.1<y<0.9$ the cross section at HERA is $\sigma_{\text {HERA }}^{(I)}=29.2_{-8.1}^{+9.9} \mathrm{pb}[$ [6, 12]. The error given is only reflecting the uncertainties of $\Lambda_{\overline{\mathrm{MS}}}$. The cut $Q^{2}>113 \mathrm{GeV}^{2}$ has not yet been included in the experimental analysis.

\footnotetext{
${ }^{1}$ The pseudo-rapidity $\eta$ is defined as $\eta=-\log \tan (\theta / 2), \theta$ being the polar angle and the proton direction being the positive $z$-axis.
} 

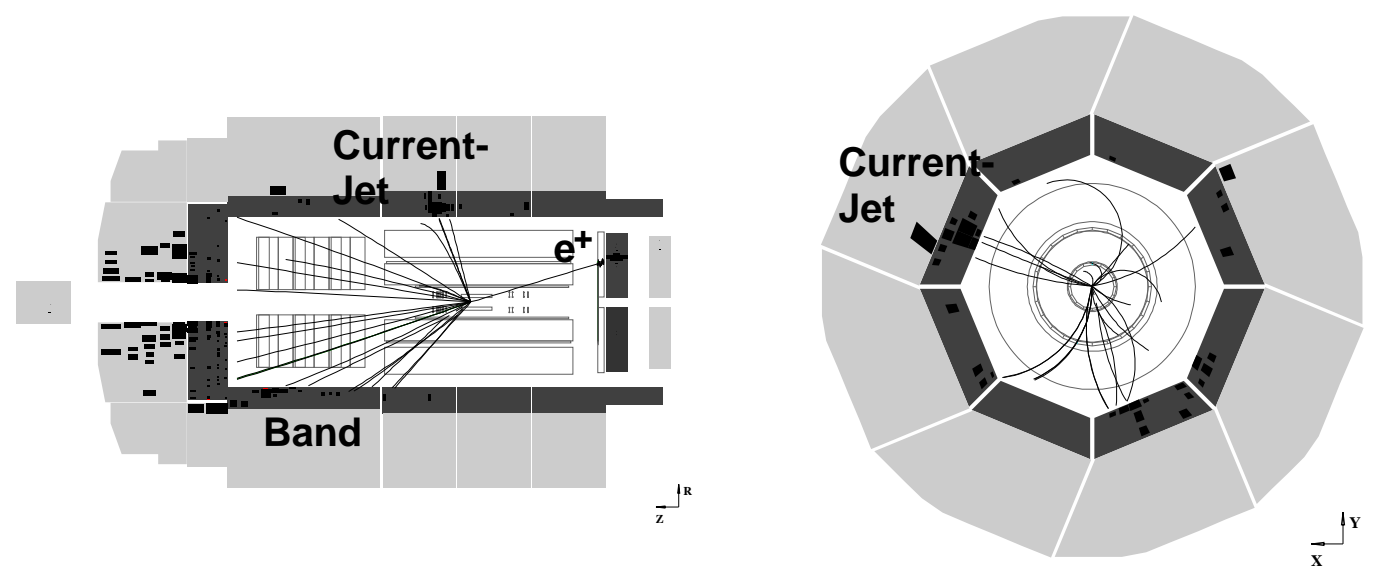

Figure 2: Example of a simulated instanton event at $Q^{2}=54 \mathrm{GeV}^{2}, x=$ $2.97 \cdot 10^{-3}, Q^{\prime 2}=136.5 \mathrm{GeV}^{2}$ and $x^{\prime}=0.48$ in the $\mathrm{H} 1$ detector. The incoming proton is moving in $+z$-direction.

\section{QCD Monte Carlo Generators}

The instanton induced processes described above are simulated by the Monte Carlo event generator QCDINS [13, 14], which works on top of the HERWIG 19 generator and calculates the matrix element of the hard subprocess. The default implementation of QCDINS is used with $x^{\prime}>0.35, Q^{\prime 2}>113 \mathrm{GeV}^{2}$. After the hard subprocess has been generated, gluons are emitted according to the leading logarithm approximation. The hadronization step describing the transition of the outgoing partons to hadrons is done using cluster fragmentation [20].

A detailed simulation of the hadronic final state of the background DIS events is provided by the two Monte Carlo event generators RAPGAP [21] and ARIADNE [22]. Both generators incorporate the $\mathcal{O}\left(\alpha_{s}\right)$ matrix elements. The main difference is the treatment of gluon emission where RAPGAP implements the parton shower approach 23] while ARIADNE employs the colour dipole model [24]. We will therefore refer to RAPGAP as "MEPS" and ARIADNE as "CDM" in the following.

\section{Event Selection}

The data were collected in the year 1997 when the HERA machine collided $E_{e}=27.5 \mathrm{GeV}$ positrons with $E_{p}=820 \mathrm{GeV}$ protons. The accumulated data sample recorded with the $\mathrm{H} 1$ detector amounted to an integrated luminosity of $15.8 \mathrm{pb}^{-1}$. A detailed description of the $\mathrm{H} 1$ detector can be found in [25]. 
A scematic view of a simulated QCDINS event in the H1 detector is given in figure 2. For this analysis the important detector components are the backward SpaCal calorimeter which is necessary for the measurement of the scattered positron and the liquid argon (LAr) calorimeter which together with the tracking system measures the particles in the $I$-band and the current jet. Because the positron is only identified using the backward SpaCal calorimeter polar angles are limited to $156^{\circ}<\theta_{e}<176^{\circ}$ which restricts the analysis to values of $Q^{2}<100 \mathrm{GeV}^{2}$.

\section{$5 \quad$ Finding discriminating variables}

The most characteristic feature of $I$-induced events is the densely populated band of hadrons in the laboratory frame due to the isotropic decay of the instanton in its rest system. Therefore the measurement of the energy flow of the hadronic final state is crucial to the identification of $I$-induced events. The energy flow in the detector is reconstructed combining the calorimetric energy depositions in the LAr and SpaCal calorimeters with the momenta of low momentum tracks $\left(p_{t}<2 \mathrm{GeV}\right)$ in the central jet chamber according to the procedure described in [26]. Also the second striking feature of $I$-induced events, the current jet, relies on this measurement.

From a theoretical point of view the most interesting observables characteristic to $I$-induced events are $x^{\prime}$ and $Q^{\prime 2}$ since they are linked to the instanton size and distance which show a characteristic behaviour. Therefore reconstructing these kinematic variables of the $I$-subprocess from the hadronic final state is paramount to identifying $I$-induced events.

The starting point of the reconstruction of the underlying kinematics can either be finding the current jet or reconstructing the expected band of hadrons in the detector. Both ways have been intensively studied [27] and a preference to identifying the band first was given. The first step is to reconstruct the mean pseudo-rapidity $\bar{\eta}$ of the band by finding the $E_{t}$ weighted mean $\eta$ of the calorimeter clusters

$$
\bar{\eta}=\frac{1}{\sum E_{t}} \sum_{\text {clusters }} \eta E_{t}
$$

and define the band by $\eta_{B} \in[\bar{\eta}-1.1, \bar{\eta}+1.1]$. This spread of 1.1 rapidity units of the band is expected from basic theoretical considerations.

The second step is to find the current jet which has roughly an $E_{t}$ of $5 \mathrm{GeV}$, as can be seen in figure 1 . Using the CONE jet algorithm with a cone radius of $R=\sqrt{\left(\eta^{2}+\phi^{2}\right)}=0.5$ allows to identify the jet in about $70 \%$ of the cases. The reason for the relatively small cone size is the fact that 

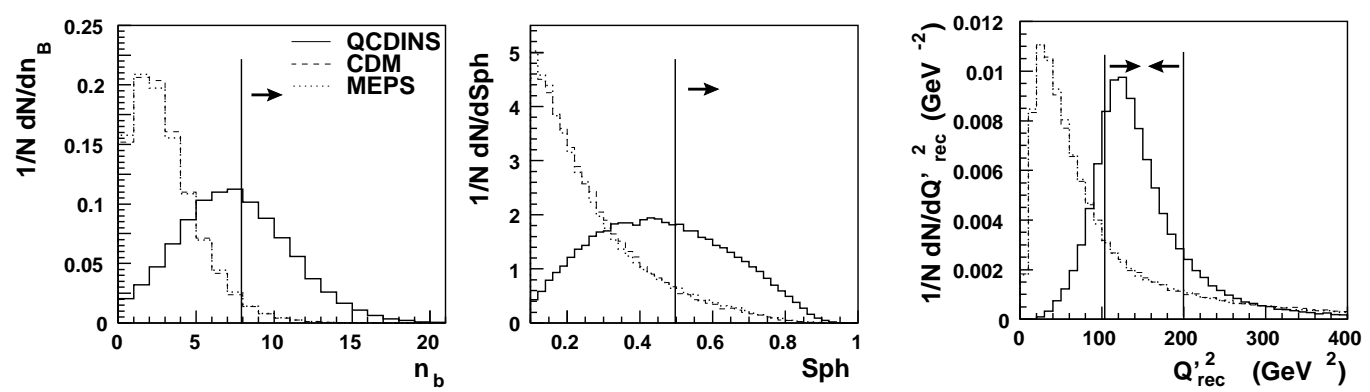

Figure 3: The shape normalized distributions of three variables which allow discriminating between standard DIS background and I-induced events. Shown are the number of charged particles in the $I$-band $n_{\mathrm{b}}$, the sphericity Sph and the reconstructed virtuality of the quark entering the $I$-subprocess $Q_{\text {rec }}^{\prime 2}$. The lines and the associated arrows denote the cuts applied.

the correct current jet can also be directed into the $I$-band and thus a bigger cone radius is prone to pick up more energy from the band while the cone size is still sufficient to find jets and reconstruct their kinematics reliably when they are well separated from the band. If the jet is emitted into the same $\eta$ and $\phi$ region as the band, then the clusters belonging to the jet are removed from the band. After the reconstruction of the jet $Q^{\prime 2}$ can be calculated and assuming the invariant mass of the band to be the mass $W_{I}$ of the instanton allows to derive $x^{\prime}$ (see figure 11).

The tagged band can now also be exploited to calculate properties that should be characteristic to the particles produced by an instanton transition. That is a high isotropy of the outgoing particles, a high number of charged particles which can be seen in the tracking system of the detector and possibly an increased strangeness production.

For this analysis the following three variables have been chosen to characterize the $I$-induced events: The reconstructed virtuality of the quark entering the $I$-subprocess $Q_{\text {rec }}^{\prime 2}$, the number of charged particles in the $\eta$-region of the band $n_{b}$ and the sphericity of all particles except those belonging to the jet, calculated in their centre of mass frame. These variables are shown in figure 3. Because of their potential in discriminating I-induced events from background and due to the good description of the data prior to any cuts these variables are used in a cut procedure to enhance the $I$-signal over the DIS signal. The distributions are generally described within $5 \%$ by the standard DIS Monte Carlo programs except for the tails which are only described within $10 \%$ (for the highest multiplicities up to $20 \%$ ).

Three additional observables have been chosen which are used to monitor the remaining events after the cuts and to see whether they support the 

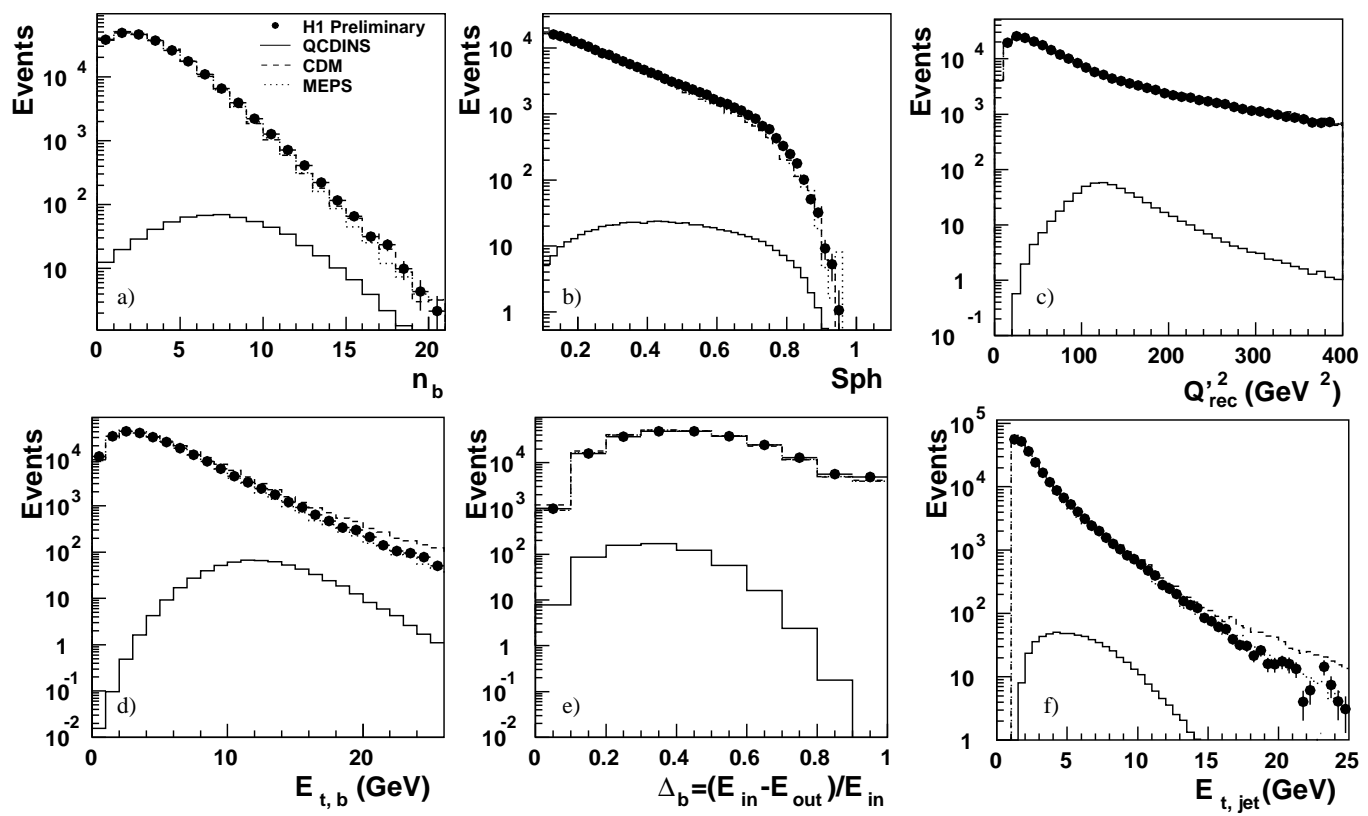

Figure 4: The distributions of three variables which are used to discriminate between standard DIS background and $I$-induced events are shown in plots a) - c). They are the number of charged particles in the $I$-band $n_{\mathrm{b}}$, the sphericity Sph and the reconstructed virtuality of the quark entering the $I$ subprocess $Q_{\mathrm{rec}}^{\prime 2}$. Plots d)-f) show the transverse energy in the $I$-band $E_{\mathrm{t}, \mathrm{b}}$, the isotropy variable $\Delta_{\mathrm{b}}$ and the transverse energy of the supposed current jet.

I-hypothesis after after discriminating against the DIS background. The variables are the total transverse energy of the band $E_{\mathrm{t}, \mathrm{b}}$, the isotropy variable $\Delta_{b}$ described below and calculated for the band and the transverse energy of the jet $E_{\mathrm{t}, \mathrm{jet}}$. These variables are described not as well as the first three, but still the description differs by about only $10 \%$.

The isotropy variable of the band $\Delta=\left(E_{\text {in,b }}-E_{\text {out }, \mathrm{b}}\right) / E_{\mathrm{in}, \mathrm{b}}$ is defined using the quantities

$$
E_{\text {out }}=\min _{\vec{i}} \sum_{n \text { Hadr. }}\left|\vec{p}_{n} \cdot \vec{i}\right| \quad E_{\text {in }}=\max _{\vec{i}} \sum_{n \text { Hadr. }}\left|\vec{p}_{n} \cdot \vec{i}\right|
$$

where $E_{\text {out }}$ will be small for a non-isotropic set of hadrons since then there is a direction $\vec{i}$ onto which the projections of the hadron momenta $\vec{p}_{n}$ is small and $E_{\text {in }}$ will be large for the same set of non-isotropically distributed hadrons. Therefore $\Delta$ is approximately unity for a non-isotropic distribution and vanishes for isotropic distributions. 


\section{$6 \quad$ Search Strategy and Results}

In order to reduce the standard DIS background, several different combinations of cuts into the three observables $Q_{\text {rec }}^{\prime 2}$, Sph and $n_{b}$ are studied and evaluated for their ability to separate $I$-induced events from the background by achieving a high separation power $S=\epsilon_{\mathrm{INS}} / \epsilon_{\mathrm{DIS}}$, where $\epsilon$ is the fraction of events left after the cuts. 125 different scenarios where surveyed which represent all possible combinations of five different values at which to cut into the three observables. The following cuts are used: $n_{b}>5,6,7,8,9, \mathrm{Sph}>$ 0.4, 0.5, 0.55, 0.6, 0.65 and for $Q_{\mathrm{rec}}^{\prime 2}: 95,100,105,110,115<Q_{\mathrm{rec}}^{\prime 2}<200 \mathrm{GeV}^{2}$. Three different scenarios were singled out according to the following criteria:

(A) The highest instanton efficiency $\left(\epsilon_{\mathrm{INS}}\right)$

(B) High $\epsilon_{\mathrm{INS}}$ at reasonable background reduction $\left(\epsilon_{\mathrm{DIS}}\right)$

(C) Highest separation power $S$ at $\epsilon_{\mathrm{INS}} \approx 10 \%$

The values of the cuts chosen in the three scenarios as well as the number of events in the data and the two standard DIS Monte Carlo simulators is shown in table 1. The error on the number of MC events includes systematical and statistical uncertainties. The systematic experimental uncertainties are of an order of 5-10\% and the two available standard DIS Monte Carlo models based on leading order QCD matrix elements and implementing different treatments of higher order parton emissions agree with each other within $20 \%$. The number of data events is about $20 \%$ larger than predicted by the DIS models. Note that this is about the difference of the prediction of the two Monte Carlo generators and the background estimation is largely unknown in this extreme phase space region. Whether the excess can be explained by instanton induced processes or is simply due to an incomplete implementation of higher order parton emissions or due to another possible deficiency in the description of the DIS background, for example, an incorrect treatment of heavy quarks, remains an open question.

Looking at the three variables after the cuts of scenario C (figure 5) shows that the three variables which were used in the cut scenario would support the $I$-hypothesis while especially the distribution of $E_{\mathrm{t}, \mathrm{b}}$ and $E_{\mathrm{t}, \mathrm{Jet}}$ disfavour it. In both of these distributions data shows a softer behaviour than the DIS Monte Carlo programs while QCDINS predicts a harder $E_{\mathrm{t}}$ spectrum. Recent theoretical analysis shows, that this might be due to the lack of a cut in $Q^{2}>113 \mathrm{GeV}^{2}$ which suppresses the contribution of non-planar graphs in the $I$-subprocess and which are not implemented in QCDINS. In fact, Schrempp and Ringwald have shown that it is possible to soften the $E_{\mathrm{t}}$ 

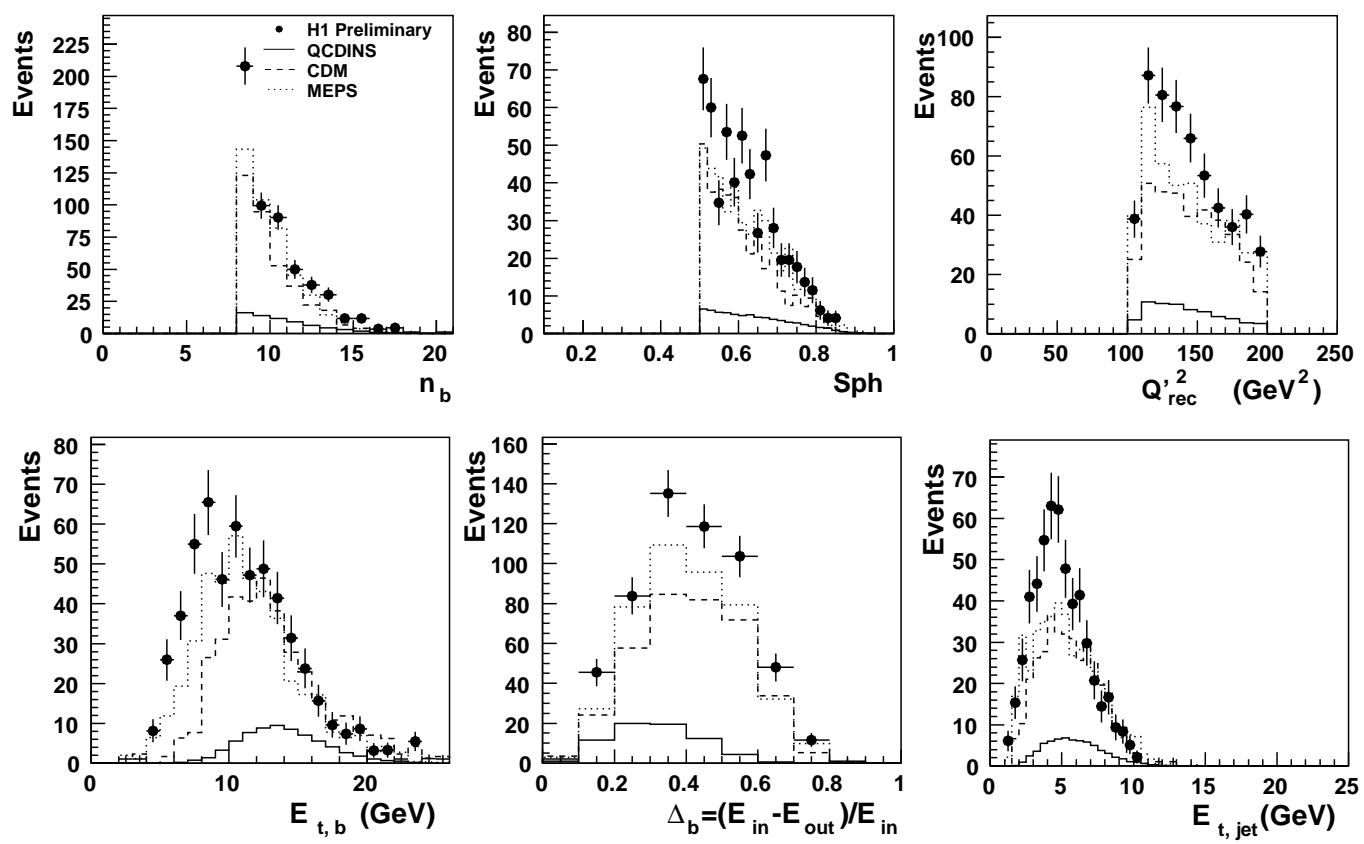

Figure 5: The six observables after the cuts to enhance the instanton signal of cut scenario C.

Table 1: The three cuts scenarios A, B and $\mathrm{C}$ which are used to reduce the background.

\begin{tabular}{|c|c|c|c|c|c|c|c|c|c|}
\hline \multicolumn{3}{|c|}{ Scenario } & $\epsilon_{\text {INS }}$ & \multicolumn{3}{|c|}{$\frac{\epsilon_{\text {INS }}}{\epsilon_{\text {IS }}}$} & \multicolumn{3}{|c|}{ \# Events } \\
& $Q^{\prime 2}\left[\mathrm{GeV}^{2}\right]$ & $S p h>$ & $n_{\mathrm{b}} \geq$ & & CDM & MEPS & CDM & MEPS & Data \\
\hline $\mathrm{A}$ & $95-200$ & 0.4 & 5 & $32 \%$ & 35 & 34 & $2469_{-238}^{+242}$ & $2572_{-222}^{+237}$ & 3000 \\
$\mathrm{~B}$ & $105-200$ & 0.4 & 7 & $21 \%$ & 56 & 52 & $1005_{-70}^{+82}$ & $1084_{-46}^{+75}$ & 1332 \\
$\mathrm{C}$ & $105-200$ & 0.5 & 8 & $11 \%$ & 86 & 71 & $363_{-26}^{+22}$ & $435_{-22}^{+36}$ & 549 \\
\hline
\end{tabular}

spectra of the jet and the band while not affecting the other four distributions by a cut against low $x$ and thus, by exploiting the strong correlation between $x$ and $Q^{2}$ in QCDINS and suppressing the contribution of events at low $Q^{2}$ [15, 16].

It will therefore be definitely interesting to extend the analysis to high $Q^{2}$ where the theoretical uncertainties in particular on these two variables are smaller. In the following section a possible search strategy is presented. 


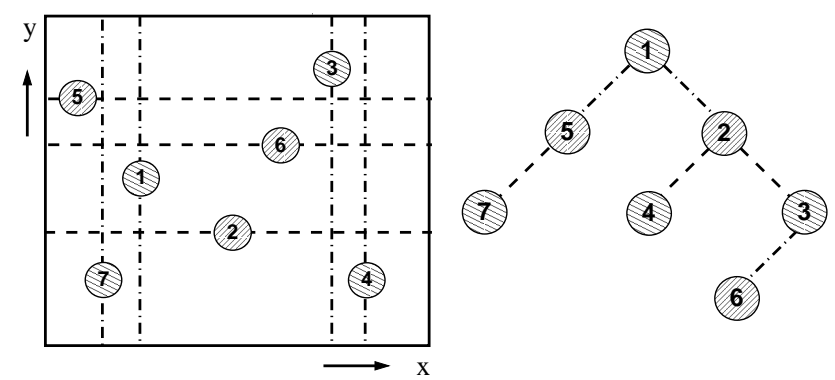

Figure 6: Example of a binary tree storing events 1-7 according to their position in the $x-y$ plane. The first event is always the top node of the tree which has two daughter nodes for events with smaller and larger $x$-value. On the second level the tree then sorts according to $y$.

\section{$7 \quad$ A Prospect of a Search at $Q^{2}>113 \mathrm{GeV}^{2}$}

Since the expected cross section of $I$-induced events in the kinematic region $\left(x_{B}>10^{-3}, 0.1<y<0.9, Q^{2}>113 \mathrm{GeV}^{2}\right)$ is by approximately two orders of magnitude lower than the DIS cross section $\sigma_{\text {DIS }} \approx 3000 \mathrm{pb}$, it is important to gain the highest possible signal to background ratio. This can be achieved by exploiting observables characterising $I$-induced processes. To find these observables a large number of promising event variables have to be investigated and the sensitivity to systematic details in the modelling of the hadronic final state has to be tested. This requires a sophisticated and fast discrimination method to find the appropriate combination of event variables. Range Searching allows to define such a powerful discriminant.

\subsection{A Discriminant Based on Range Searching}

Events can be classified as signal or background by estimating their probability density $\rho$ at the point of the event in the event-variable phase space, employing a Monte Carlo (MC) generator to sample the densities. In the case of neural networks (NN) this is done by fitting the probability-density with the adjusted weights of the neurons. To circumvent this time consuming procedure the density at each point can be directly estimated by counting the number of background and signal events in a surrounding box $V$. Given the ratio

$$
\ell:=\frac{\rho(I)}{\rho(D I S)}=\frac{\# I(V)}{\# D I S(V)}
$$


the probability of an event to be a signal event is $D=\ell /(1+\ell)$. Compared to NN's this method also has the advantage of not extrapolating into phase space regions where there are no sample events available. Thus signals from data events outside the region covered by the MC simulation can be avoided. Counting the number of events in the vicinity of a certain point is a problem known as Range Searching.

Range Searching algorithms have been developed which allow a search time $\sim \log (n)$, where $n$ is the number of points that have to be searched [17]. An algorithm using binary trees to store the events is employed, suitable especially for a large number of events and dimensions (i.e. observables). The MC events are successively filled into the nodes of two binary trees, one for the signal events and one for the background events, where the criterion by which the decision is taken to descend to the left or right of a node is given by the value of one of the event variables (see figure 6). While descending the tree this variable cycles through all considered discriminating variables. After filling, the position of every event in the tree is given by its coordinates in the event variable space. Classification of an event is done by searching in the trees for all background and signal events in the box $V$. This is done in the same manner as filling the tree. A more detailed description and the properties of this method can be found in [28.

\subsection{Results}

Starting with 35 variables based on the hadronic final state the best 12 were chosen by calculating the discriminant with all 2-combinations (pairs) of the initial variables and taking those variables which provide a high separation power $S=\epsilon_{\mathrm{INS}} / \epsilon_{\mathrm{DIS}}$ demanding an efficiency for instantons of $\epsilon_{\mathrm{INS}}=10 \%$. The number of considered variables is further reduced by calculating all 5combinations and selecting those with the highest separation power and a small systematic variation of the background. The systematic uncertainty was obtained by using four standard DIS-MC simulators which were tuned to data on representative hadronic final state quantities, in the range $Q^{2}>$ $100 \mathrm{GeV}^{2}$ at HERA [18]. The variables forming the best combination is shown in Figure 77. The separation power for $\epsilon_{\mathrm{INS}}=10 \%$ is $S=126$. In Figure 8 the shape normalized discriminant $D$ is shown for the $I$-induced and the background events, as well as the distribution for $D>0.9$ normalized to a luminosity of $100 \mathrm{pb}^{-1}$ which is comparable to that already collected by each of the HERA experiments H1 and ZEUS. An event sample can be isolated where half of the events are expected to be due to instantons while the $I$-efficiency is still $10 \%$.

In addition the reconstruction of $x^{\prime}$ and $Q^{\prime} 2$ was studied before and after 

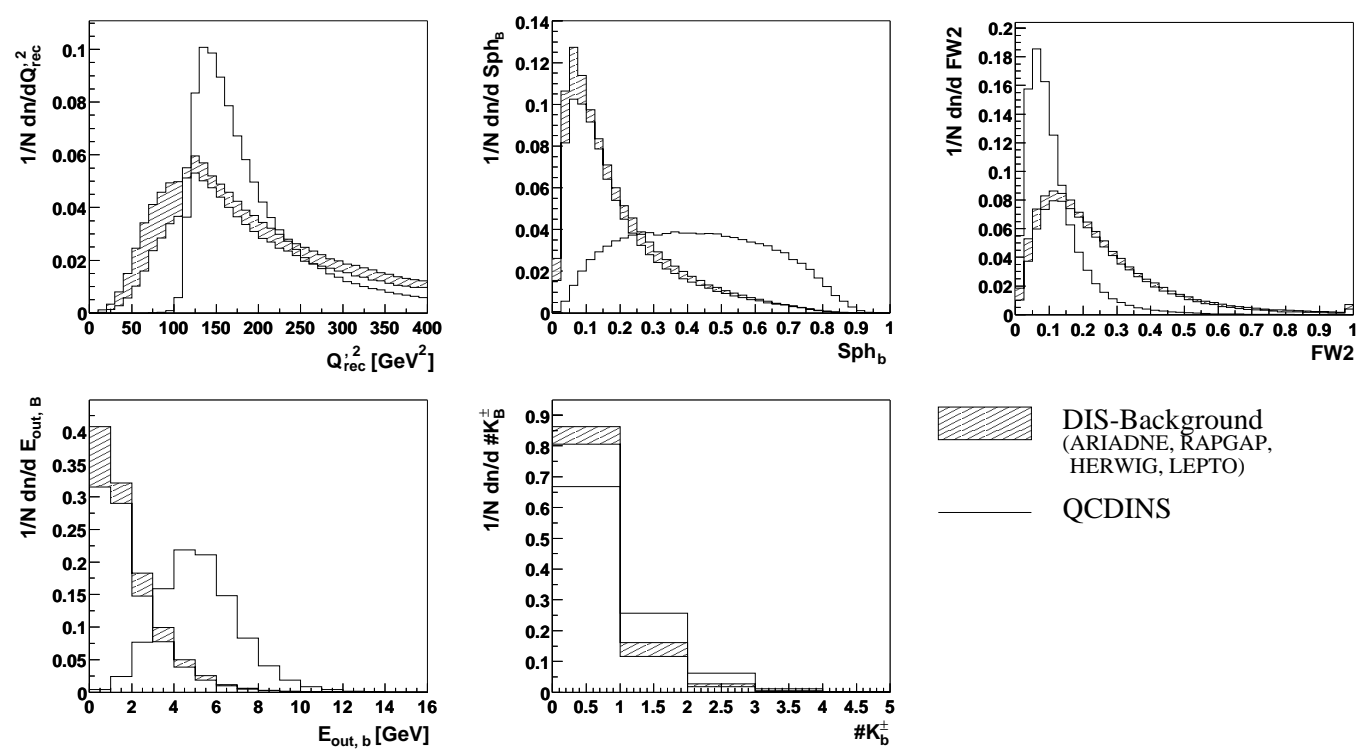

Figure 7: The characteristic event variables providing good instanton separation with small systematic uncertainties. Shown is the reconstructed virtuality of the quark entering the $I$-subprocess $Q_{\text {rec }}^{\prime 2}$, the sphericity of the particles not associated to the jet in their rest system, the second Fox-Wolfram moment of these particles and the event shape variable $E_{\text {out,B }}$ which is the projection of the particle transverse energy onto the axis, that makes this quantity maximal (see [8]). Finally the number of charged kaons in the $I$ Band is shown.

a cut on $D$. As can be seen from figure 9, a cut on the discriminant at $D>0.95$ improves the reconstruction of both variables strongly. The events which were selected by the discriminant are very I-like and therefore the reconstruction based on ideal $I$-events is successful.

\section{An Outlook on Proving Chirality Violation}

The one property of $I$-induced events which is not present in standard deep inelastic scattering is chirality violation. Therefore finding events which violate chirality would be a strong hint for $I$-induced processes.

In every $I$-induced event chirality is violated by $\Delta \chi=2 n_{f}$, meaning that all the outgoing quarks are right handed (or all left handed, in the case of anti-instantons). In addition, in every $I$-induced event a pair of $s-\bar{s}$ quarks is produced. The decay of $\Lambda$ baryons might provide a unique way of measuring the polarization of these strange quarks, provided both quarks form a $\Lambda$ 

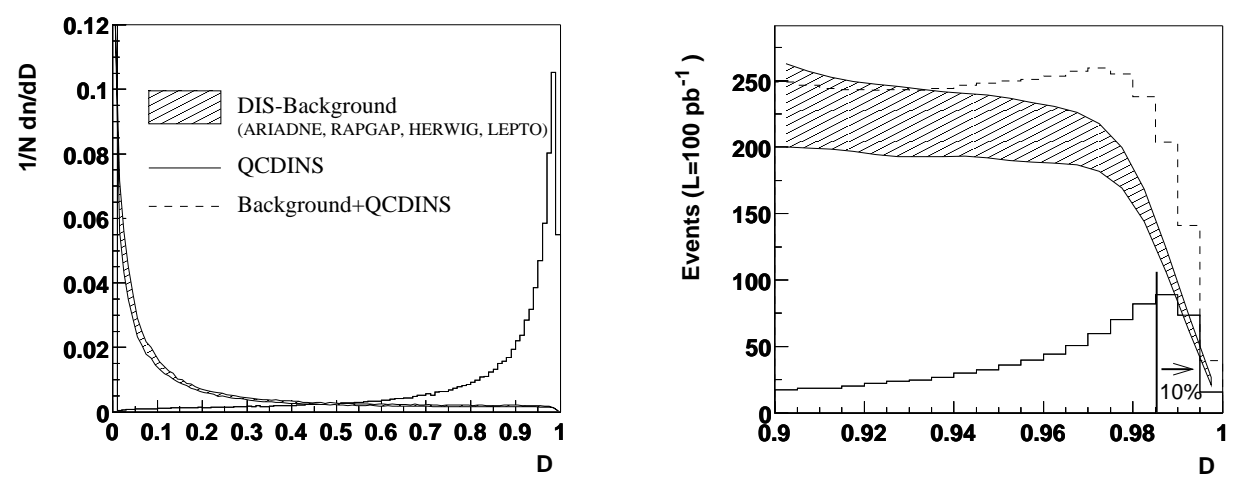

Figure 8: To the left, the shape normalized discriminant $D$ for the instanton events using QCDINS and for standard DIS events using four MC simulators is shown. The second plot shows a zoom into the rightmost part and is now normalized to a luminosity of $L=100 \mathrm{pb}^{-1}$. At $\epsilon_{\mathrm{INS}}=10 \% 178 \mathrm{I}$-induced events are expected.

and $\bar{\Lambda}$-baryons after hadronization respectively. However, the problem here is that a large fraction of the $\Lambda$-baryons contain $s$-quarks stemming from fragmentation. So far, no efficient selection mechanism has been found to select $\Lambda$-pairs containing only strange quarks produced in the hard instanton process. Inferring the polarization of the $s$-quark from the $\Lambda$-decay has been well studied in polarized deep inelastic scattering [31.

An additional problem of such an analysis is the relatively low detector efficiency for $\Lambda$-baryons which is of the order of $25 \%$ for a rapidity range of $|\eta|<1.3$ for the H1-detector, which leads to only a $6 \%$ chance of finding both $\Lambda$-baryons in an event.

\section{Conclusions}

H1 has conducted a search for $I$-induced events in the kinematic range of $x_{\mathrm{Bj}}>10^{-3}, 0.1<y<0.6$ and $\theta_{\mathrm{el}}>156^{\circ}$ using 1997 data. Using a simple cut technique the standard DIS background has been suppressed up to the $0.1 \%$ level while retaining $10 \%$ of the $I$-induced events. More events were found in the data than predicted by the DIS Monte Carlo programs. Nevertheless this excess is not conclusive since it is of the order of the difference of the Monte Carlo predictions. While four out of the six distributions that were studied would support the $I$-hypothesis after these cuts, the $E_{\mathrm{t}}$ spectra of the band and the jet do not favour it. However, recent theoretical analysis has shown that this discrepancy can be explained by the lack of a cut $Q^{2}>113 \mathrm{GeV}^{2}$ 

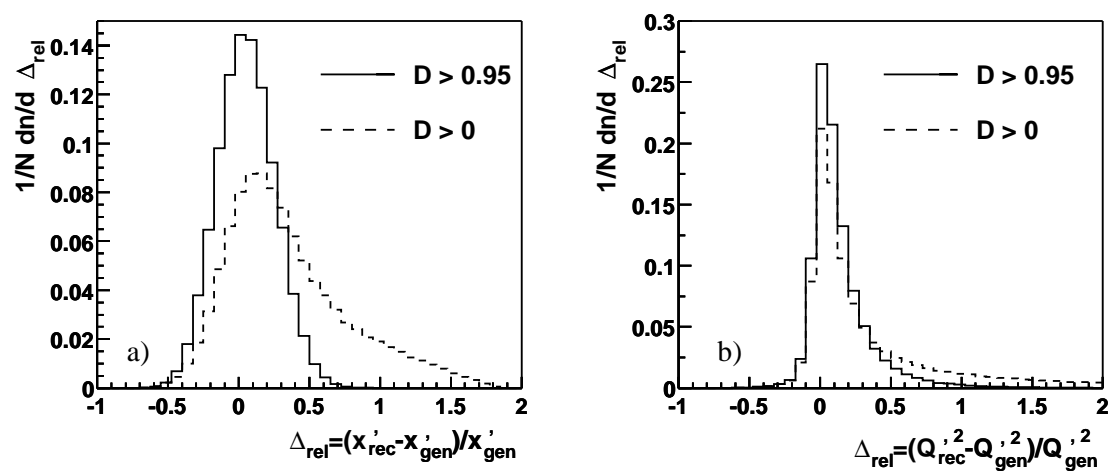

Figure 9: The reconstruction of $x^{\prime}$ and $Q^{\prime 2}$ strongly improves when a cut on the discriminator is applied. With a cut $D>0.95$ the RMS of the reconstruction of $x^{\prime}$ is 0.20 and 0.17 for $Q^{\prime 2}$.

which suppresses non-planar graphs not present in the QCDINS Monte Carlo simulator.

It is therefore of high interest to extend the analysis to this range of $Q^{2}$ to reduce the theoretical uncertainties. A first survey of the prospects of such a search was done using the Range Searching method to discriminate between the $I$-signal and the DIS background. The results are very promising since this method allows to choose combinations of observables with small systematic uncertainties in the final discriminant and a high separation power.

A possibility of measuring the chirality violation of the $I$-induced events via the decay of $\Lambda$-baryons is in principle possible if an efficient mechanism to select $\Lambda$-baryons containing only strange quarks from the hard $I$-process can be found and experimental difficulties can be overcome. In any case such an analysis will require a very large luminosity and an isolated data sample where the contamination from standard DIS processes is low.

\section{Acknowledgements}

I would like to thank A. Ringwald and F. Schrempp for many enlightening and delighting discussions about instantons and for their invaluable tool QCDINS. T. Carli and S. Mikocki I would like to thank for their fruitful collaboration in this hunt for instantons. I am very grateful to Chr. Risler for providing the information on $\Lambda$-identification, and M. zur Nedden and P. Schleper for their careful reading of this paper. 


\section{References}

[1] Belavin A et al. 1975 Phys. Lett. B 5985

[2] 't Hooft G 1976 Phys. Rev. D 143432

[3] Ringwald A and Schrempp F 1995 Proc. Int. Sem. Quarks '94 (World Scientific) Preprint hep-ph/9411217

[4] Moch S, Ringwald A and Schrempp F 1997 Nucl. Phys. B 507134

[5] Ringwald A and Schrempp F 1998 Phys. Lett. B 438217

[6] Ringwald A and Schrempp F 1999 Phys. Lett. B 459249

[7] Gibbs et al. 1996 Proc. Future Physics at HERA vol 1 p 509

[8] Carli T, Gerigk J, Ringwald A and Schrempp F 1999 Proc. Monte Carlo Generators for HERA Physics Workshop (Hamburg: Desy Proceedings) p 329 Preprint hep-ph/9906441

[9] Carli T and Kuhlen M 1998 Nucl. Phys. B 51185

[10] H1 Collaboration, S. Aid et al. 1996 Z. Phys C 72573

[11] H1 Collaboration, S. Aid et al. 1996 Nucl. Phys. B 4803

[12] Ringwald A and Schrempp F 2000 Proc. Workshop on New Trends in HERA Physics 1999 (Springer) Preprint hep-ph/9909338

[13] Gibbs M, Ringwald A and Schrempp F 1995 Proc. DIS 1995 (Paris, France) pp 341-344 Preprint hep-ph/9506392

[14] Ringwald A and Schrempp F 2000 Comp. Phys. Comm. 132267

[15] Ringwald A and Schrempp F 2001 Phys. Lett. B 503331

[16] Schrempp F 2001 Talk given at the Workshop on New Trends in HERA Physics (Ringberg, Germany)

[17] Sedgewick R 1992 Algorithms in C++ (Addison Wesley) Chapter 26

[18] Brook N et al. 1999 Proc. Workshop on Monte Carlo Generators for HERA Physics (Hamburg: Desy Proceedings) p 10

[19] Marchesini G et al. 1992 Comp. Phys. Comm. 67465 
[20] Webber B 1992 Nucl. Phys. B 238492

[21] Jung H 1995 Comp. Phys. Comm. 86147

[22] Lönnblad 1992 Comp. Phys. Comm. 7115

[23] Bengtsson M and Sjöstrand T 1998 Z. Phys. C 37465

[24] Gustafson G and Petterson U 1988 Nucl. Phys. B 306 741; Gustafson G 1986 Phys. Lett. B 175 453; Andersson B, Gustafson G, Lönnblad L and Petterson U 1989 Z. phys. C 43625

[25] H1 Collaboration, Abt I et al. 1997 Nucl. Instr. and Meth. A 386310

[26] H1 Collaboration, Adloff C et al. 2000 Eur. Phys. J. C 313609

[27] Gerigk J 1998 Dipl. Thesis University of Hamburg and MPI-PhE/98-20

[28] Koblitz B and Carli T Proc. Advanced Comp. Analysis Techniques in Physics Research 2000 Preprint hep/ph0011224

[29] Mikocki S for the H1 collaboration, 2000 Int. Workshop on DIS 2000 (Liverpool, United Kingdom) Preprint hep-ex/007008

[30] H1 Collaboration Int. Conf. on High Energy Physics 2000, (Osaka, Japan)

[31] Ma B Q, Schmidt I, Soffer J and Yang J J 2000 Eur. Phys. J. C 16657 